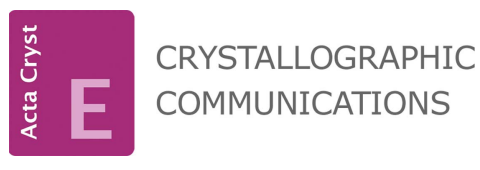

ISSN 2056-9890

Received 8 July 2019

Accepted 18 July 2019

Edited by M. Weil, Vienna University of Technology, Austria

Keywords: crystal structure; halogen bonds; hydrogen bonds; solvate.

CCDC reference: 1941436

Supporting information: this article has supporting information at journals.iucr.org/e

\section{Crystal structure and Hirshfeld surface analysis of 2,5-dibromoterephthalic acid ethylene glycol monosolvate}

\author{
Kenika Khotchasanthong, ${ }^{a}$ Siripak Jittirattanakun, ${ }^{\mathrm{b}}$ Suwadee Jiajaroen, ${ }^{\mathrm{b}}$ Chatphorn \\ Theppitak $^{b}$ and Kittipong Chainok ${ }^{\mathrm{a} *}$
}

\begin{abstract}
aMaterials and Textile Technology, Faculty of Science and Technology, Thammasat University, Khlong Luang, Pathum Thani, 12121, Thailand, and ${ }^{\mathbf{b}}$ Division of Chemistry, Faculty of Science and Technology, Thammasat University, Khlong Luang, Pathum Thani, 12121, Thailand. *Correspondence e-mail: kc@tu.ac.th
\end{abstract}

The title compound, $\mathrm{C}_{8} \mathrm{H}_{4} \mathrm{Br}_{2} \mathrm{O}_{4} \cdot \mathrm{C}_{2} \mathrm{H}_{6} \mathrm{O}_{2}$, crystallizes with one-half of a 2,5dibromoterephthalic acid $\left(\mathrm{H}_{2} \mathrm{Br}_{2} \mathrm{tp}\right)$ molecule and one-half of an ethylene glycol (EG) molecule in the the asymmetric unit. The whole molecules are generated by application of inversion symmetry. The $\mathrm{H}_{2} \mathrm{Br}_{2}$ tp molecule is not planar, with the dibromobenzene ring system inclined by a dihedral angle of $18.62(3)^{\circ}$ to the carboxylic group. In the crystal, the $\mathrm{H}_{2} \mathrm{Br}_{2}$ tp and EG molecules are linked into sheets propagating parallel to $(\overline{101)}$ through $\mathrm{O}-\mathrm{H} \cdots \mathrm{O}$ hydrogen bonds, thereby forming $R_{4}^{4}(12)$ and $R_{4}^{4}$ (28) graph-set motifs. $\mathrm{Br} \cdots \mathrm{O}$ and weak $\pi-\pi$ stacking interactions are also observed. Hirshfeld surface analysis was used to confirm the existence of these interactions.

\section{Chemical context}

Terephthalic acid and its derivatives are important ligands in the construction of coordination frameworks with high dimensionalities and interesting topologies ( $\mathrm{Li}$ et al., 1999; Seidel et al., 2011). They have also been shown to be versatile building blocks in crystal engineering to drive the selfassembly of functional supramolecular networks through intermolecular interactions such as hydrogen bonds, halogen bonds, and aromatic $\pi-\pi$ stacking interactions (Lemmerer, 2011; Karmakar et al., 2014; Meng et al., 2015).

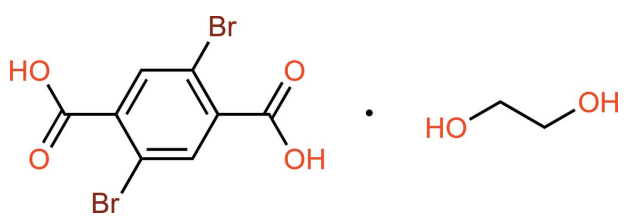

In this study, we present the crystal structure of 2,5-dibromoterephthalic acid ethylene glycol monosolvate, $\mathrm{C}_{8} \mathrm{H}_{4} \mathrm{Br}_{2} \mathrm{O}_{4} \cdot \mathrm{C}_{2} \mathrm{H}_{6} \mathrm{O}_{2}$ or $\mathrm{H}_{2} \mathrm{Br}_{2} \mathrm{tp} \cdot \mathrm{EG}$, which is a pseudopolymorph of the previously reported compound 2,5-dibromoterephthalic acid dihydrate (Song et al., 2008).

\section{Structural commentary}

The structures of the molecular components in the title compound are shown in Fig. 1. The asymmetric unit contains one-half of $\mathrm{a}_{2} \mathrm{Br}_{2}$ tp molecule and one-half of an EG molecule. Both molecules are generated by application of inversion symmetry. The $\mathrm{H}_{2} \mathrm{Br}_{2} \mathrm{tp}$ molecule is not planar. Its 
Table 1

Hydrogen-bond geometry $\left(\AA,^{\circ}\right)$.

\begin{tabular}{lllll}
\hline$D-\mathrm{H} \cdots A$ & $D-\mathrm{H}$ & $\mathrm{H} \cdots A$ & $D \cdots A$ & $D-\mathrm{H} \cdots A$ \\
\hline $\mathrm{O} 1-\mathrm{H} 1 \cdots \mathrm{O} 3$ & $0.83(1)$ & $1.75(1)$ & $2.559(3)$ & $165(4)$ \\
$\mathrm{O} 3-\mathrm{H} 3 \cdots \mathrm{O} 2{ }^{\mathrm{i}}$ & $0.82(1)$ & $1.97(1)$ & $2.767(3)$ & $166(4)$ \\
\hline
\end{tabular}

Symmetry code: (i) $-x+2,-y+1,-z+2$.

dibromobenzene ring system (r.m.s. deviation $=0.006 \AA$ ) makes a dihedral angle of $18.62(3)^{\circ}$ with the carboxylic group (r.m.s. deviation $=0.013 \AA$ ). As a result of symmetry restrictions, the EG molecule adopts an anti-conformation with an $\mathrm{O} 3-\mathrm{C} 5-\mathrm{C}^{\mathrm{i}}-\mathrm{O}^{\mathrm{i}}$ torsion angle of $180^{\circ}$ [symmetry code: (i) $2-x,-y, 2-z]$.

\section{Supramolecular features}

In the crystal, the $\mathrm{H}_{2} \mathrm{Br}_{2}$ tp and EG molecules are linked by strong-to-medium $\mathrm{O}-\mathrm{H} \cdots \mathrm{O}$ hydrogen bonds between carboxylic acid and alcohol $\mathrm{OH}$ functions (Table 1), enclosing $R_{4}^{4}$ (12) and $R_{4}^{4}$ (28) graph-set motifs and forming sheets parallel to the (101) plane; Fig. 2 . Br $\cdots \mathrm{O}$ halogen bonding $[\mathrm{Br} \cdots \mathrm{O}=$ 3.2536 (4) $\left.\AA ; \mathrm{C}-\mathrm{Br} \cdots \mathrm{O}=157.7(3)^{\circ}\right]$ and weak $\pi-\pi$ stacking interactions [centroid-to-centroid distance $=4.283(5) \AA$ ] are also observed (Fig. 3). The combination of these intermolecular interactions results in the formation of a threedimensional supramolecular network.

\section{Hirshfeld surface analysis}

Hirshfeld surfaces (McKinnon et al., 2007) and their associated two-dimensional fingerprint plots (Spackman \& McKinnon, 2002) were used to quantify the various intermolecular interactions, and were generated using CrystalExplorer17 (Turner et al., 2017). The shorter and longer contacts are indicated as red and blue spots on the Hirshfeld surfaces, and contacts with distances equal to the sum of the van der Waals radii are represented as white spots. Hirshfeld surfaces of the title compound mapped over the normalized distance, $d_{\text {norm }}$, using a standard surface resolution with a fixed

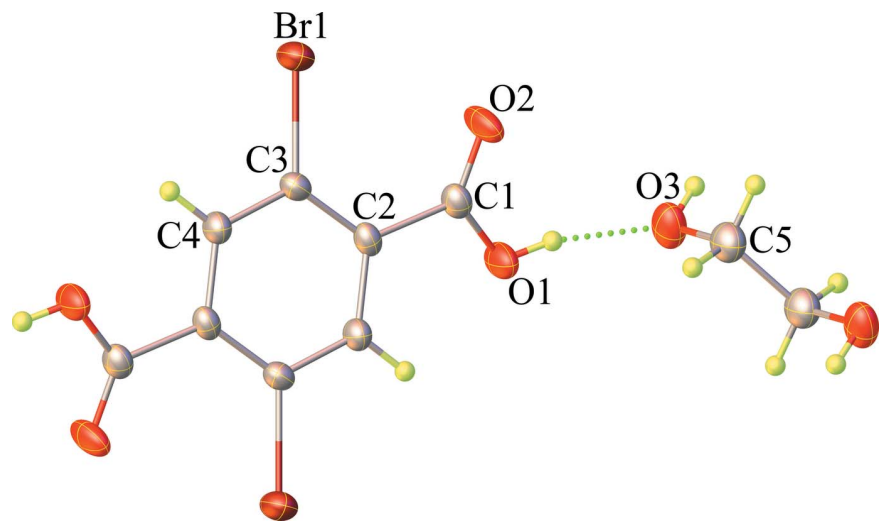

Figure 1

The structures of the molecular components in the title compound with displacement ellipsoids drawn at the $50 \%$ probability level. The $\mathrm{O}-$ $\mathrm{H}$. . O hydrogen bond is shown by a dashed line.

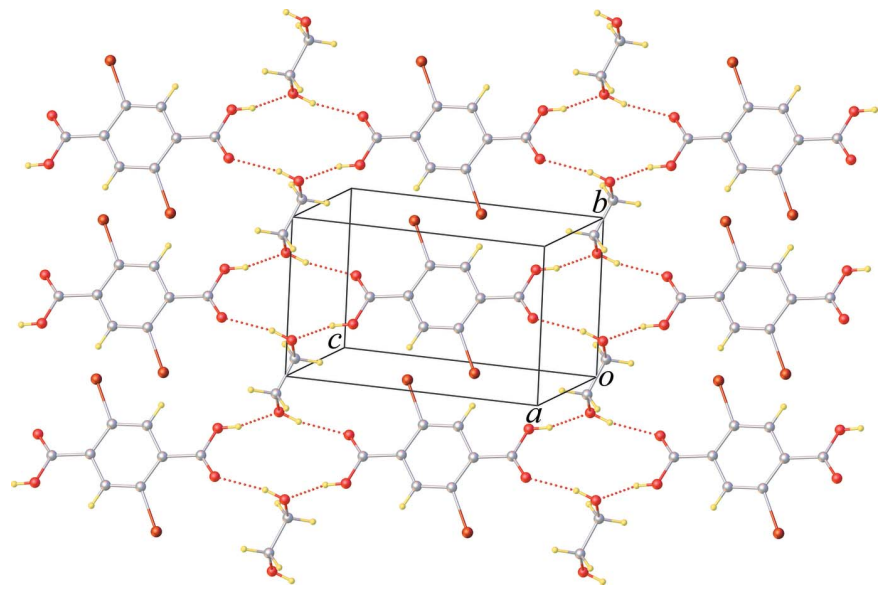

Figure 2

View of a supramolecular two-dimensional sheet parallel to the (101) direction, enclosing $R_{4}^{4}$ (12) and $R_{4}^{4}$ (28) graph-set motifs, sustained by $\mathrm{O}-\mathrm{H} \cdots \mathrm{O}$ hydrogen bonds (dashed lines).

colour scale of -0.7877 (red) to 0.9385 a.u. (blue) and the twodimensional fingerprint plots are illustrated in Fig. 4. The dominant interactions between $\mathrm{H}$ and $\mathrm{O}$ atoms, corresponding to the discussed $\mathrm{O}-\mathrm{H}$... O hydrogen bonds, can be clearly be seen as red spots on the Hirshfeld surface. The faint-red spot visible on the $d_{\text {norm }}$ surface can be assigned to Br...O contacts. Analysis of the two-dimensional fingerprint plots reveals that the $\mathrm{H} \cdots \mathrm{O} / \mathrm{O} \cdots \mathrm{H}(28.8 \%)$ contacts are the dominant contributors to the Hirshfeld surface. The contribution of the $\mathrm{Br} \cdots \mathrm{H} / \mathrm{H} \cdots \mathrm{Br}$ contacts is $22.1 \%$, whereas $\mathrm{Br} \cdots \mathrm{Br}$ contacts are negligible $(0.9 \%)$. Other contacts viz. $\mathrm{H} \cdot \mathrm{H}(17.7 \%)$, $\mathrm{H} \cdots \mathrm{C} / \mathrm{C} \cdots \mathrm{H}(7.7 \%), \mathrm{Br} \cdots \mathrm{C} / \mathrm{C} \cdots \mathrm{Br}(7.2 \%), \mathrm{Br} \cdots \mathrm{O} / \mathrm{O} \cdots \mathrm{Br}$ $(5.8 \%), \mathrm{C} \cdots \mathrm{O} / \mathrm{O} \cdots \mathrm{C}(4.5 \%), \mathrm{C} \cdots \mathrm{C}(3.3 \%)$ and $\mathrm{O} \cdots \mathrm{O}$ $(2.2 \%)$ also make significant contributions to the Hirshfeld surface.

\section{Database survey}

A search of the Cambridge Structural Database (Version 5.40, latest update May 2019; Groom et al., 2016) for the $\mathrm{H}_{2} \mathrm{Br}_{2} \mathrm{tp}$ entity resulted in just two matches. In the structure of the pseudopolymorphic $\mathrm{H}_{2} \mathrm{Br}_{2} \mathrm{tp}$ dihydrate (CSD refcode POFROS; Song et al., 2008), the $\mathrm{H}_{2} \mathrm{Br}_{2} \mathrm{tp}$ molecules are connected through water molecules by $\mathrm{O}-\mathrm{H} \cdots \mathrm{O}$ hydrogen bonds, forming a three-dimensional supramolecular network.

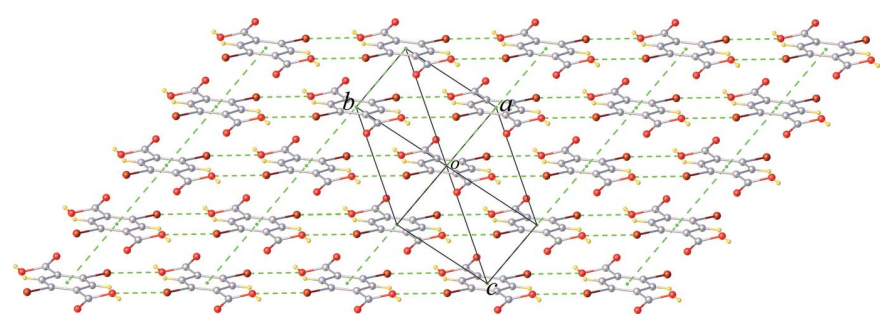

Figure 3

View along [111] of a supramolecular sheet sustained by $\mathrm{Br} \cdots \mathrm{O}$ halogen bonding and $\pi-\pi$ stacking interactions (dashed lines). 


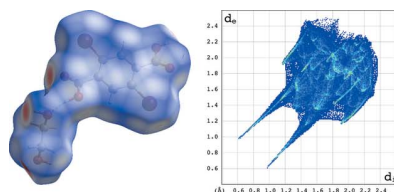

(a) All $\cdots$ All

(c) $\mathrm{H} \cdots \mathrm{Br} / \mathrm{Br} \cdots \mathrm{H}(21.1 \%)$

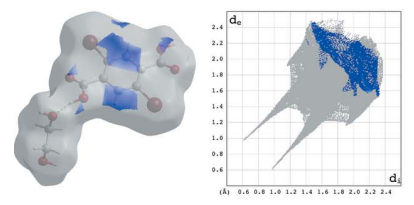

(e) $\mathrm{H} \cdots \mathrm{C} / \mathrm{C} \cdots \mathrm{H}(7.7 \%)$

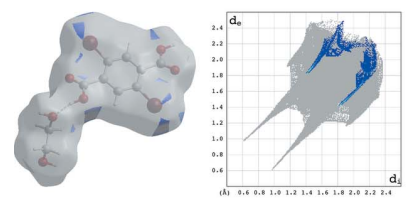

(g) $\mathrm{Br} \cdots \mathrm{O} / \mathrm{O} \cdots \mathrm{Br}(5.8 \%)$

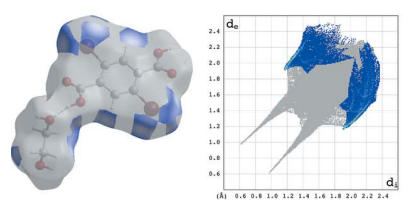

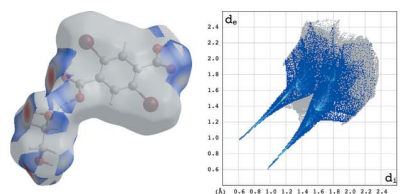

(b) $\mathrm{H} \cdots \mathrm{O} / \mathrm{O} \cdots \mathrm{H}(28.8 \%)$

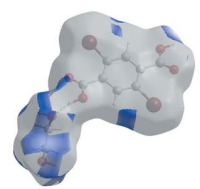

(d) $\mathrm{H} \cdots \mathrm{H}(17.7 \%)$

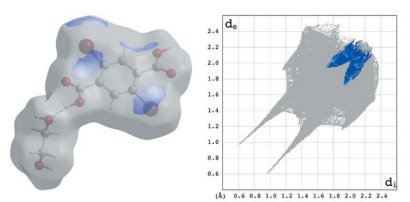

(f) $\mathrm{Br} \cdots \mathrm{C} / \mathrm{C} \cdots \mathrm{Br}(7.2 \%)$

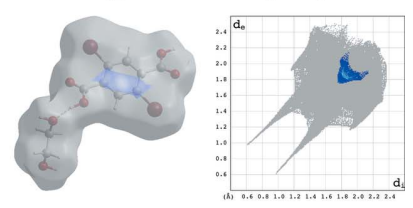

(h) $\mathrm{C} \cdots \mathrm{C}(3.3 \%)$
Figure 4

Two-dimensional fingerprint plots of the title compound, showing $(a)$ all interactions, and delineated into $(b) \mathrm{H} \cdots \mathrm{O} / \mathrm{O} \cdots \mathrm{H},(c) \mathrm{H} \cdots \mathrm{Br} / \mathrm{Br} \cdots \mathrm{H}$, (d) $\mathrm{H} \cdots \mathrm{H},(e) \mathrm{H} \cdots \mathrm{C} / \mathrm{C} \cdots \mathrm{H},(f) \mathrm{H} \cdots \mathrm{O} / \mathrm{O} \cdots \mathrm{H},(g) \mathrm{Br} \cdots \mathrm{O} / \mathrm{O} \cdots \mathrm{Br}$, and (h) C $\cdots \mathrm{C}$ interactions $\left[d_{\mathrm{e}}\right.$ and $d_{\mathrm{i}}$ represent the distances from a point on the Hirshfeld surface to the nearest atoms outside (external) and inside (internal) the surface, respectively].

In the structure of bis $\{N$-[1-(pyridin-2-yl- $\kappa N)$ ethylidene]pyridine-4-carbohydrazonato- $\kappa^{2} N^{\prime}, O$ \}nickel(II)-2,5-dibromoterephthalic acid (OBOJEX; Nakanishi \& Sato, 2017), the $\mathrm{H}_{2} \mathrm{Br}_{2}$ tp molecules form hydrogen-bonded zigzag chains with the complex molecules. The packing is further consolidated by $\pi-\pi$ stacking and $\mathrm{Br} \cdots \mathrm{Br}$ halogen bonding.

\section{Synthesis and crystallization}

$\mathrm{H}_{2} \mathrm{Br}_{2}$ tp and EG were purchased from commercial sources and used as received. A solution of $\mathrm{H}_{2} \mathrm{Br}_{2} \mathrm{tp}(0.020 \mathrm{~g})$ in $5 \mathrm{ml}$ of EG was heated ( $333 \mathrm{~K}$ ) to reflux for $15 \mathrm{~min}$. The reaction solution was held for $2-3 \mathrm{~h}$ and colourless block-shaped crystals suitable for single-crystal X-ray diffraction analysis were obtained.

\section{Refinement}

Crystal data, data collection and structure refinement details are summarized in Table 2. The carbon-bound $\mathrm{H}$ atoms were placed in geometrically calculated positions and refined as riding with $\mathrm{C}-\mathrm{H}=0.93 \AA$ for aromatic and $\mathrm{C}-\mathrm{H}=0.97 \AA$ for methylene hydrogen atoms with $U_{\text {iso }}(\mathrm{H})=1.2 U_{\text {eq }}(\mathrm{C})$. The $\mathrm{H}$ atoms bound to $\mathrm{O}$ atoms were located from difference-Fourier maps but were refined with distance restraints of $\mathrm{O}-\mathrm{H}=0.82$ $\pm 0.02 \AA$ and $U_{\text {iso }}(\mathrm{H})=1.5 U_{\text {eq }}(\mathrm{O})$.
Table 2

Experimental details.

Crystal data

Chemical formula

$M_{\mathrm{r}}$

Crystal system, space group

Temperature (K)

$a, b, c(\AA)$

$\alpha, \beta, \gamma\left({ }^{\circ}\right)$

$V\left(\AA^{3}\right)$

$Z$

Radiation type

$\mu\left(\mathrm{mm}^{-1}\right)$

Crystal size (mm)

$\mathrm{C}_{8} \mathrm{H}_{4} \mathrm{Br}_{2} \mathrm{O}_{4} \cdot \mathrm{C}_{2} \mathrm{H}_{6} \mathrm{O}_{2}$

386.00

Triclinic, $P \overline{1}$

296

4.2823 (6), 6.2607 (9), 11.5497 (17)

96.701 (5), 93.930 (5), 90.575 (5)

$306.76(8)$

1

Mo $K \alpha$

6.62

$0.20 \times 0.20 \times 0.16$

Data collection

Diffractometer

Absorption correction

$T_{\min }, T_{\max }$

No. of measured, independent and observed $[I>2 \sigma(I)]$ reflections

$R_{\text {int }}$

$(\sin \theta / \lambda)_{\max }\left(\AA^{-1}\right)$

Bruker D8 QUEST CMOS
PHOTON II
Multi-scan (SADABS; Bruker,
2016)
$0.576,0.747$
$9075,1208,1076$
0.052
0.617

$0.023,0.052,1.05$
1208
91
2
H atoms treated by a mixture of
$\quad$ independent and constrained
$\quad$ refinement
$0.34,-0.28$

Refinement

$R\left[F^{2}>2 \sigma\left(F^{2}\right)\right], w R\left(F^{2}\right), S$

No. of reflections

No. of parameters

No. of restraints

$\mathrm{H}$-atom treatment

$\Delta \rho_{\max }, \Delta \rho_{\min }\left(\mathrm{e} \AA^{-3}\right)$ $0.34,-0.28$

Computer programs: APEX3 and SAINT (Bruker, 2016), SHELXT (Sheldrick, 2015a), SHELXL2014 (Sheldrick, $2015 b$ and OLEX2 (Dolomanov et al., 2009).

\section{Acknowledgements}

The authors thank the Faculty of Science and Technology, Thammasat University, for funds to purchase the X-ray diffractometer.

\section{Funding information}

This work was supported by a National Research Councils of Thailand grant provided by the Thammasat University (No. 20/2561).

\section{References}

Bruker (2016). APEX3, SAINT and SADABS. Bruker AXS Inc., Madison, Wisconsin, USA.

Dolomanov, O. V., Bourhis, L. J., Gildea, R. J., Howard, J. A. K. \& Puschmann, H. (2009). J. Appl. Cryst. 42, 339-341.

Groom, C. R., Bruno, I. J., Lightfoot, M. P. \& Ward, S. C. (2016). Acta Cryst. B72, 171-179.

Karmakar, A., Oliver, C. L., Platero-Prats, A. E., Laurila, E. \& Öhrström, L. (2014). CrystEngComm, 16, 8243-8251.

Lemmerer, A. (2011). Cryst. Growth Des. 11, 583-593.

Li, H., Eddaoudi, M., O'Keeffe, M. \& Yaghi, O. M. (1999). Nature, 402, 276-279.

McKinnon, J. J., Jayatilaka, D. \& Spackman, M. A. (2007). Chem. Commun. pp. 3814-3816.

Meng, F., Li, Y., Liu, X., Li, B. \& Wang, L. (2015). Cryst. Growth Des. 15, 4518-4525.

Nakanishi, T. \& Sato, O. (2017). Acta Cryst. E73, 103-106. 
Seidel, C., Ahlers, R. \& Ruschewitz, U. (2011). Cryst. Growth Des. 11, 5053-5063.

Sheldrick, G. M. (2015a). Acta Cryst. A71, 3-8.

Sheldrick, G. M. (2015b). Acta Cryst. C71, 3-8.

Song, G.-L., Liu, S., Liu, H.-J., Zeng, T. \& Zhu, H.-J. (2008). Acta Cryst. E64, o1860.
Spackman, M. A. \& McKinnon, J. J. (2002). CrystEngComm, 4, 378392.

Turner, M. J., Mckinnon, J. J., Wolff, S. K., Grimwood, D. J., Spackman, P. R., Jayatilaka, D. \& Spackman, M. A. (2017). CrystalExplorer17. The University of Western Australia. 


\section{supporting information}

Acta Cryst. (2019). E75, 1228-1231［https://doi.org/10.1107/S2056989019010260]

\section{Crystal structure and Hirshfeld surface analysis of 2,5-dibromoterephthalic acid ethylene glycol monosolvate}

\section{Kenika Khotchasanthong, Siripak Jittirattanakun, Suwadee Jiajaroen, Chatphorn Theppitak and Kittipong Chainok}

\section{Computing details}

Data collection: APEX3 (Bruker, 2016); cell refinement: SAINT (Bruker, 2016); data reduction: SAINT (Bruker, 2016); program(s) used to solve structure: SHELXT (Sheldrick, 2015a); program(s) used to refine structure: SHELXL2014 (Sheldrick, 2015b); molecular graphics: OLEX2 (Dolomanov et al., 2009); software used to prepare material for publication: OLEX2 (Dolomanov et al., 2009).

(I)

\section{Crystal data}

$\mathrm{C}_{8} \mathrm{H}_{4} \mathrm{Br}_{2} \mathrm{O}_{4} \cdot \mathrm{C}_{2} \mathrm{H}_{6} \mathrm{O}_{2}$

$M_{r}=386.00$

Triclinic, $P \overline{1}$

$a=4.2823(6) \AA$

$b=6.2607$ (9) $\AA$

$c=11.5497(17) \AA$

$\alpha=96.701(5)^{\circ}$

$\beta=93.930(5)^{\circ}$

$\gamma=90.575(5)^{\circ}$

$V=306.76(8) \AA^{3}$

$$
\begin{aligned}
& Z=1 \\
& F(000)=188 \\
& D_{\mathrm{x}}=2.089 \mathrm{Mg} \mathrm{m}^{-3}
\end{aligned}
$$

Mo $K \alpha$ radiation, $\lambda=0.71073 \AA$

Cell parameters from 5153 reflections

$\theta=3.3-28.8^{\circ}$

$\mu=6.62 \mathrm{~mm}^{-1}$

$T=296 \mathrm{~K}$

Block, light colourless

$0.20 \times 0.20 \times 0.16 \mathrm{~mm}$

\section{Data collection}

Bruker D8 QUEST CMOS PHOTON II diffractometer

Radiation source: sealed x-ray tube, Micro focus tube

Graphite monochromator

Detector resolution: 7.39 pixels $\mathrm{mm}^{-1}$

$\omega$ and $\varphi$ scans

Absorption correction: multi-scan

(SADABS; Bruker, 2016)

\section{Refinement}

Refinement on $F^{2}$

Least-squares matrix: full

$R\left[F^{2}>2 \sigma\left(F^{2}\right)\right]=0.023$

$w R\left(F^{2}\right)=0.052$

$S=1.05$

1208 reflections
$T_{\min }=0.576, T_{\max }=0.747$

9075 measured reflections

1208 independent reflections

1076 reflections with $I>2 \sigma(I)$

$R_{\text {int }}=0.052$

$\theta_{\max }=26.0^{\circ}, \theta_{\min }=3.3^{\circ}$

$h=-5 \rightarrow 5$

$k=-7 \rightarrow 7$

$l=-14 \rightarrow 14$

91 parameters

2 restraints

Primary atom site location: dual

Hydrogen site location: mixed

$\mathrm{H}$ atoms treated by a mixture of independent and constrained refinement 
$w=1 /\left[\sigma^{2}\left(F_{\mathrm{o}}^{2}\right)+(0.0175 P)^{2}+0.2228 P\right]$

where $P=\left(F_{\mathrm{o}}^{2}+2 F_{\mathrm{c}}^{2}\right) / 3$

$(\Delta / \sigma)_{\max }<0.001$

$\Delta \rho_{\max }=0.34$ e $\AA^{-3}$

$\Delta \rho_{\min }=-0.28$ e $\AA^{-3}$

Special details

Geometry. All esds (except the esd in the dihedral angle between two 1.s. planes) are estimated using the full covariance matrix. The cell esds are taken into account individually in the estimation of esds in distances, angles and torsion angles; correlations between esds in cell parameters are only used when they are defined by crystal symmetry. An approximate (isotropic) treatment of cell esds is used for estimating esds involving l.s. planes.

Fractional atomic coordinates and isotropic or equivalent isotropic displacement parameters $\left(\AA^{2}\right)$

\begin{tabular}{lllll}
\hline & $x$ & $y$ & $z$ & $U_{\text {iso }} * U_{\text {eq }}$ \\
\hline Br1 & $0.28840(7)$ & $0.90781(4)$ & $0.67985(2)$ & $0.03714(15)$ \\
O1 & $0.9576(5)$ & $0.3651(3)$ & $0.75182(16)$ & $0.0399(5)$ \\
H1 & $1.013(9)$ & $0.352(6)$ & $0.8205(14)$ & $0.071(12)^{*}$ \\
O2 & $0.6124(5)$ & $0.5932(3)$ & $0.82669(15)$ & $0.0430(5)$ \\
O3 & $1.1223(5)$ & $0.2556(3)$ & $0.95346(17)$ & $0.0415(5)$ \\
H3 & $1.205(8)$ & $0.320(5)$ & $1.0136(19)$ & $0.065(12)^{*}$ \\
C1 & $0.7228(6)$ & $0.4945(4)$ & $0.7436(2)$ & $0.0263(5)$ \\
C2 & $0.6046(5)$ & $0.5048(4)$ & $0.6187(2)$ & $0.0231(5)$ \\
C3 & $0.4170(6)$ & $0.6668(4)$ & $0.5809(2)$ & $0.0244(5)$ \\
C4 & $0.3155(6)$ & $0.6610(4)$ & $0.4647(2)$ & $0.0261(5)$ \\
H4 & 0.190106 & 0.770860 & 0.441344 & $0.031^{*}$ \\
C5 & $0.9128(7)$ & $0.0972(4)$ & $0.9857(2)$ & $0.0371(6)$ \\
H5A & 0.756819 & 0.056821 & 0.921709 & $0.044^{*}$ \\
H5B & 0.805174 & 0.156980 & 1.052949 & $0.044^{*}$ \\
\end{tabular}

Atomic displacement parameters $\left(\AA^{2}\right)$

\begin{tabular}{lllllll}
\hline & $U^{11}$ & $U^{22}$ & $U^{33}$ & $U^{12}$ & $U^{13}$ & $U^{23}$ \\
\hline Br1 & $0.0530(2)$ & $0.03148(18)$ & $0.02537(18)$ & $0.01196(12)$ & $0.00214(11)$ & $-0.00380(10)$ \\
O1 & $0.0505(12)$ & $0.0469(12)$ & $0.0215(10)$ & $0.0198(9)$ & $-0.0049(9)$ & $0.0034(9)$ \\
O2 & $0.0507(12)$ & $0.0585(13)$ & $0.0175(9)$ & $0.0199(10)$ & $-0.0031(8)$ & $-0.0034(9)$ \\
O3 & $0.0535(13)$ & $0.0409(11)$ & $0.0290(11)$ & $-0.0047(10)$ & $-0.0110(10)$ & $0.0079(9)$ \\
C1 & $0.0292(13)$ & $0.0285(13)$ & $0.0206(12)$ & $-0.0021(10)$ & $-0.0026(10)$ & $0.0029(10)$ \\
C2 & $0.0261(12)$ & $0.0261(12)$ & $0.0170(11)$ & $-0.0032(10)$ & $-0.0007(9)$ & $0.0037(9)$ \\
C3 & $0.0299(13)$ & $0.0239(12)$ & $0.0189(11)$ & $0.0015(10)$ & $0.0031(10)$ & $-0.0006(9)$ \\
C4 & $0.0307(13)$ & $0.0272(12)$ & $0.0204(12)$ & $0.0050(10)$ & $-0.0011(10)$ & $0.0044(10)$ \\
C5 & $0.0386(15)$ & $0.0414(15)$ & $0.0310(14)$ & $0.0065(12)$ & $-0.0030(12)$ & $0.0062(12)$ \\
\hline
\end{tabular}

Geometric parameters $\left(\stackrel{A}{\circ}{ }^{\circ}\right)$

\begin{tabular}{llll}
\hline $\mathrm{Br} 1-\mathrm{C} 3$ & $1.894(2)$ & $\mathrm{C} 2-\mathrm{C} 3$ & $1.392(3)$ \\
$\mathrm{O} 1-\mathrm{H} 1$ & $0.825(10)$ & $\mathrm{C} 2-\mathrm{C} 4^{\mathrm{i}}$ & $1.394(3)$ \\
$\mathrm{O} 1-\mathrm{C} 1$ & $1.303(3)$ & $\mathrm{C} 3-\mathrm{C} 4$ & $1.377(3)$ \\
$\mathrm{O} 2-\mathrm{C} 1$ & $1.207(3)$ & $\mathrm{C} 4-\mathrm{H} 4$ & 0.9300 \\
$\mathrm{O} 3-\mathrm{H} 3$ & $0.817(10)$ & $\mathrm{C} 5-\mathrm{C} 5^{\mathrm{ii}}$ & $1.493(5)$
\end{tabular}




$\begin{array}{llll}\mathrm{O} 3-\mathrm{C} 5 & 1.428(4) & \mathrm{C} 5-\mathrm{H} 5 \mathrm{~A} & 0.9700 \\ \mathrm{C} 1-\mathrm{C} 2 & 1.504(3) & \mathrm{C} 5-\mathrm{H} 5 \mathrm{~B} & 0.9700 \\ \mathrm{C} 1-\mathrm{O} 1-\mathrm{H} 1 & 112(3) & \mathrm{C} 4-\mathrm{C} 3-\mathrm{C} 2 & 120.5(2) \\ \mathrm{C} 5-\mathrm{O} 3-\mathrm{H} 3 & 108(3) & \mathrm{C} 2-\mathrm{C} 4-\mathrm{H} 4 & 119.1 \\ \mathrm{O} 1-\mathrm{C} 1-\mathrm{C} 2 & 112.1(2) & \mathrm{C} 3-\mathrm{C} 4-\mathrm{C} 2^{\mathrm{i}} & 121.9(2) \\ \mathrm{O} 2-\mathrm{C} 1-\mathrm{O} 1 & 123.7(2) & \mathrm{C} 3-\mathrm{C} 4-\mathrm{H} 4 & 119.1 \\ \mathrm{O} 2-\mathrm{C} 1-\mathrm{C} 2 & 124.1(2) & \mathrm{O} 3-\mathrm{C} 5-\mathrm{C} 5^{\mathrm{ii}} & 110.6(3) \\ \mathrm{C} 3-\mathrm{C} 2-\mathrm{C} 1 & 124.6(2) & \mathrm{O} 3-\mathrm{C} 5-\mathrm{H} 5 \mathrm{~A} & 109.5 \\ \mathrm{C} 3-\mathrm{C} 2-\mathrm{C} 4^{\mathrm{i}} & 117.6(2) & \mathrm{O} 3-\mathrm{C} 5-\mathrm{H} 5 \mathrm{~B} & 109.5 \\ \mathrm{C} 4-\mathrm{C} 2-\mathrm{C} 1 & 117.8(2) & \mathrm{C} 5^{\mathrm{ii}}-\mathrm{C} 5-\mathrm{H} 5 \mathrm{~A} & 109.5 \\ \mathrm{C} 2-\mathrm{C} 3-\mathrm{Br} 1 & 124.11(18) & \mathrm{C} 5^{\mathrm{ii}}-\mathrm{C} 5-\mathrm{H} 5 \mathrm{~B} & 108.1 \\ \mathrm{C} 4-\mathrm{C} 3-\mathrm{Br} 1 & 115.39(18) & \mathrm{H} 5 \mathrm{~A}-\mathrm{C} 5-\mathrm{H} 5 \mathrm{~B} & \\ & & & \\ \mathrm{O} 3-\mathrm{C} 5-\mathrm{C} 5^{\mathrm{ii}-\mathrm{O} 3}{ }^{\mathrm{ii}} & 180.000(1) & & \end{array}$

Symmetry codes: (i) $-x+1,-y+1,-z+1$; (ii) $-x+2,-y,-z+2$.

Hydrogen-bond geometry $\left(\AA,{ }^{\circ}\right)$

\begin{tabular}{lllll}
\hline$D-\mathrm{H} \cdots A$ & $D-\mathrm{H}$ & $\mathrm{H} \cdots A$ & $D \cdots A$ & $D-\mathrm{H} \cdots A$ \\
\hline $\mathrm{O} 1-\mathrm{H} 1 \cdots \mathrm{O} 3$ & $0.83(1)$ & $1.75(1)$ & $2.559(3)$ & $165(4)$ \\
$\mathrm{O} 3-\mathrm{H} 3 \cdots \mathrm{O} 22^{\mathrm{iii}}$ & $0.82(1)$ & $1.97(1)$ & $2.767(3)$ & $166(4)$ \\
\hline
\end{tabular}

Symmetry code: (iii) $-x+2,-y+1,-z+2$. 